\title{
A computational model of nuclear self-organisation in syncytial embryos.
}

\author{
Christoph Koke ${ }^{\mathrm{a}, \mathrm{b}}$, Takuma Kanesaki ${ }^{\mathrm{c}}$, Jörg Grosshans ${ }^{\mathrm{c}}$, Ulrich S. \\ Schwarz $^{\mathrm{a}, \mathrm{b}}$, Carina M. Dunlop ${ }^{\mathrm{a}, \mathrm{d}}$ \\ ${ }^{a}$ Bioquant, University of Heidelberg, Heidelberg, Germany. \\ ${ }^{b}$ Institute for Theoretical Physics, University of Heidelberg, Heidelberg, Germany. \\ ${ }^{c}$ Institute for Biochemistry and Molecular Cell Biology, University of Göttingen, \\ Göttingen, Germany. \\ ${ }^{d}$ Department of Mathematics, University of Surrey, Guildford, UK.
}

\begin{abstract}
Syncytial embryos develop through cycles of nuclear division and rearrangement within a common cytoplasm. A paradigm example is Drosophila melanogaster in which nuclei form an ordered array in the embryo surface over cell cycles 10-13. This ordering process is assumed to be essential for subsequent cellularization. Using quantitative tissue analysis, it has previously been shown that the regrowth of actin and microtubule networks after nuclear division generate reordering forces that counteract its disordering effect (Kanesaki et al., Integrative Biology 2011). We present here an individual-based computer simulation modelling the nuclear dynamics. In contrast to similar modelling approaches for e.g. epithelial monolayers or tumour spheroids, we focus not on the spatial dependence, but rather on the time-dependence of the interaction laws. We show that appropriate phenomenological inter-nuclear force laws reproduce the experimentally observed dynamics provided that the cytoskeletal network regrows sufficiently quickly after mitosis. Then repulsive forces provided by the actin system are necessary and sufficient to regain the observed level of order in the system, after the strong disruption resulting from cytoskeletal network disassembly and spindle formation. We also observe little mixing of nuclei through cell cycles. Our study highlights the importance of the dynamics of cytoskeletal forces during this critical phase of syncytial development and emphasises the need for real-time experimental data at high temporal resolution.
\end{abstract}

Keywords: 
Cytoskeleton, Drosophila melanogaster, morphogenesis, individual-based model

\section{Introduction}

Spatial control of subcellular components is essential for cellular functionality, most obviously during e.g. mitosis or cell polarisation (Fletcher and Mullins (2010); Bornens (2008)). Such spatial control is particularly significant in multi-nucleate cells such as muscle myofibres (Metzger et al. (2012)) and invertebrate syncytial embryos (Foe and Alberts (1983)). The paradigmatical example of a syncytial embryo is Drosophila melanogaster, in which nuclei initially undergo rapid cycles of almost synchronous division and rearrangement within a common cytoplasm. These cycles begin with 9 nuclear divisions in the yolk centre (each 8 minutes long) after which the majority of the nuclei migrate to the egg surface to form a two-dimensional array at the embryo surface where they undergo a final four divisions (which take 9, 10, 12 and 21 minutes, respectively) (Foe and Alberts (1983)) (see Fig. 1). During these final four divisions the nuclear movements are constrained to the embryo surface, with the nuclei attached to the plasma membrane by an actin-based mechanism. The establishment of a regular arrangement of nuclei at the embryo surface is assumed to be essential for controlling cell size at cellularization, during which membranes form separating the nuclei into individual cells (Blankenship and Wieschaus (2001)). Additionally, regular positioning and uniform cell size are hypothesised to be important for establishing morphogen gradients within the embryo (Bollenbach et al. (2008)), and for planar cell polarity signalling later in development (Ma et al. (2008)).

To achieve organelle organisation at the subcellular level requires the action of mechanical forces. These forces are generated through the cellular cytoskeleton, which in general is formed from intermediate filaments, actin filaments and microtubules and their associated accessory proteins and motor proteins (Fletcher and Mullins (2010)). Within the syncytial embryo these cytoskeletal elements form large networks that span the embryo, potentially enabling the generation of both short- and long-range forces. In particular each nucleus in the surface array is associated with an F-actin 'cap', a pair of centrosomes and an enclosing microtubule basket, with additional cortical F-actin and astral microtubules underlying the enclosing membrane of the egg, see Fig. 1(a) (Schejter and Wieschaus (1993); Foe et al. (1993); Cao et al. (2010); Foe et al. (2000)). These structures maintain the nuclei at 
(a)
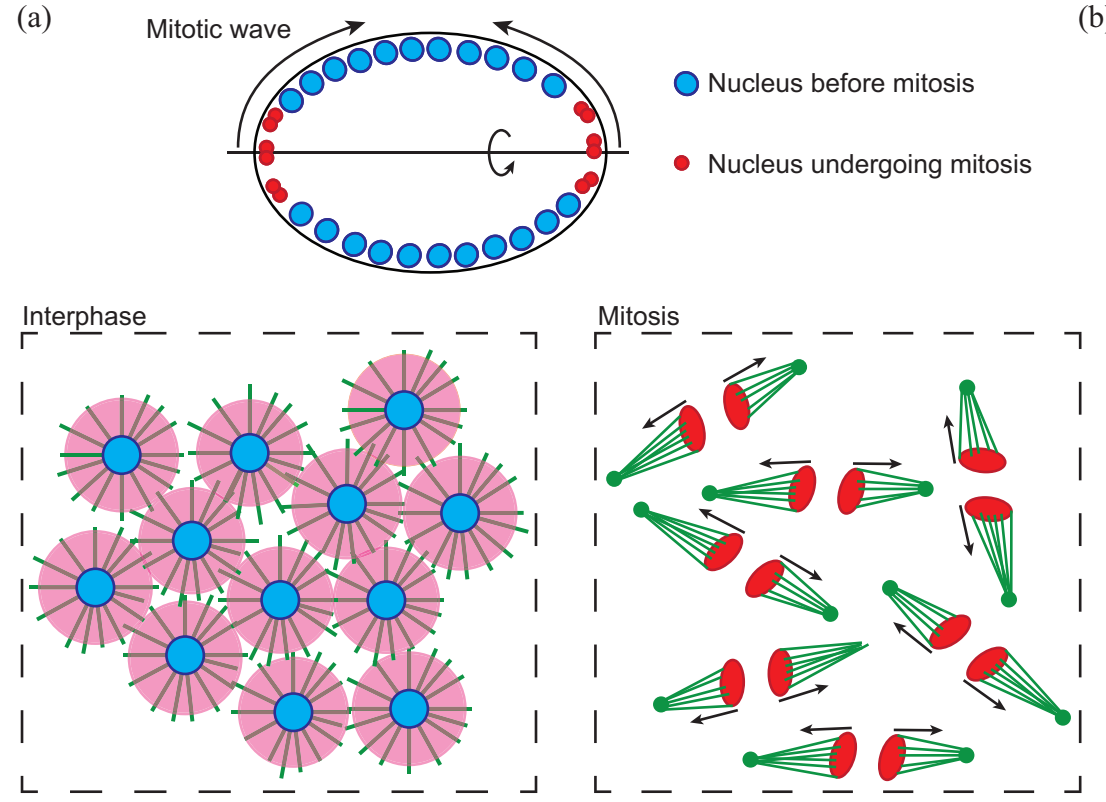

(b)

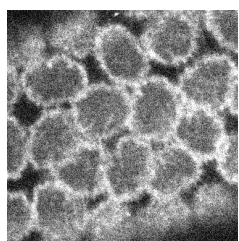

Figure 1: The syncytial embryo. (a) Schematic of a section of the syncytial embryo in which nuclei arrange themselves in a two-dimensional array at the surface. Mitosis progresses in a wave-like manner across the embryo, usually beginning at the poles. The insets are top-down views of the nuclear array showing the arrangement of the cytoskeleton during interphase and during mitosis. In interphase each nucleus is associated with a microtubule array (green lines) that emanates from its centrosomes and additionally with an actin cap that lies above it (red discs). In mitosis the cytoskeletal networks break down, with the remaining microtubules forming the mitotic spindle that drives the daughter nuclei apart. Arrows indicate the direction of force on each nucleus. (Colour online). (b) Fluorescence images showing the organisation of actin caps, nuclei and microtubules in a syncytial Drosophila melanogaster embryo during interphase 12. Top: F-actin labelled by moesin-GFP. Middle: Nuclei labelled with Histone H2Av-GFP. Scale bar $10 \mu \mathrm{m}$. Bottom: Dynamic microtubules visualised by a temporal projection of EB1-GFP images. (Reproduced from (Kanesaki et al. (2011)) by permission of The Royal Society of Chemistry.) Scale bar $10 \mu \mathrm{m}$. 
the embryo surface, while during interphase it is observed that both the actin caps and the microtubule baskets and asters overlap and interact (Foe et al. (2000)) (see Fig. 1(b)). In a similar manner to the actin caps, nuclear positioning could also be influenced by membrane-based furrows that are associated with each nucleus (Foe et al. (1993); Mazumdar and Mazumdar (2002)). However, at present it is not clear exactly how these membrane invaginations mechanically contribute to nuclear ordering.

Importantly, the cytoskeletal networks are highly dynamic. Prior to mitosis they completely break down before the mitotic spindle drives the separating daughter nuclei apart. The actin and microtubule systems then reform after division. Interestingly although nuclear division happens almost simultaneously there is in fact a small temporal offset so that division events progressively occur across the embryo. This results in the visually arresting division wave phenomenon, usually referred to as the mitotic wave (Foe and Alberts (1983)) (see video S1). The underlying mechanisms driving this phenomenon are not clear. An obvious possibility is the diffusion of a molecule involved in cell cycle control, which then triggers mitosis, leading to an excited wave based on a biochemical activator. Recently an alternative has been suggested, namely an excited wave based on mechanical stimulation (Idema et al. (2013)). The wave is predominantly initiated at the anterior and posterior poles and then travels towards the equator of the embryo within half a minute (Foe and Alberts (1983)).

We develop here an individual-based simulation of the syncytium, which enables the analysis of individual nuclear positioning throughout the cell cycle. This is the first such simulation of this system. This framework enables us to investigate the necessary conditions for cytoskeletal derived forces to achieve the observed nuclear self-organisation in the embryo. In particular, we focus for the first time on the essential time-dependence of the internuclear forces on the cell cycle. In syncytial embryos, individual nuclei are able to interact with each other directly through the cytoskeleton thus generating directly internuclear forces. As a result, the appropriate modelling framework is to use individual-based 'cell-centre' models consisting of particles interacting via position-dependent force laws, see e.g. Meineke et al. (2001); Mirams et al. (2013); Drasdo and Höhme (2005).

Various interaction potentials have been used with such cell-centre models in order to capture different cellular behaviours. These range from simple linear spring-like force laws, e.g. Mirams et al. (2013); Meineke et al. (2001), to models that include cell-cell adhesion forces (Drasdo and Höhme (2005)) and 
more complex elastic interactions, e.g. interacting elastic spheres (Schaller and Meyer-Hermann (2005)). An additional benefit of using centre-based models is the ability to coarse-grain these simulations to continuum descriptions, thus generating multi-scale models with the attendant computational advantages of continuum modelling (Drasdo (2005); Murray et al. (2012)). Individual-based models have clear advantages for modelling the syncytium as they capture the essential nature of a system. However, in contrast to the models discussed above, our focus here is on how the interaction forces change in time, rather than on the exact form of their spatial dependence. We show that a simple constant pressure model of internuclear forces can reproduce the experimentally observed nuclear ordering dynamics. This is based on a comparison with a recent experimental study that used quantitative image analysis and the calculation of time-dependent order parameters to track nuclear ordering throughout the cell cycle (Kanesaki et al. (2011)).

We predict that repulsive forces are necessary and sufficient to generate the observed reordering within the embryo. Similar ordering processes are known from densely packed colloidal systems or electrons on a sphere (Bowick et al. (2002)), but in our case the ordering occurs in a density-independent manner and after anisotropic perturbations caused by the mitotic spindles. Our results also suggest the existence of attractive forces in the cytoskeletal network associated with the microtubule network. Most importantly, we show that correct reordering of the nuclei is crucially dependent on the time of reconnection of the cytoskeletal network, and predict that it is essential that cytoskeletal networks rapidly reform after nuclear division. Disordering of the nuclei and the mitotic spindle force are found to be nonlinearly correlated, with the maximum disorder observed in the system determined by the strength of the mitotic spindle. We also observe that nuclei maintain their relative spatial positions across phases of division and rearrangement in agreement with recent 3D imaging data (Krzic et al. (2012)).

\section{Computational model of the syncytium}

We here develop an individual-based simulation of the syncytial embryo of Drosophila melanogaster. We use this simulation to investigate the ordering dynamics of the nuclei of the syncytial embryo throughout cell cycles. We only consider the final cycles during which the nuclei are constrained to move on the surface of the embryo and focus on the role of the temporal changes in the cytoskeletal networks on this process. We adopt an individual-based 
model representing each nucleus by its centre of mass $\mathbf{x}_{i}$ and its nuclear age $\tau_{i} \in\left[0, \tau_{c}\right]$, where $\tau_{c}$ is the cell cycle length. Internuclear forces are calculated based on time since division. Significantly very different forcegenerating mechanisms act during nuclear division and during the periods between divisions and so the model is split into two parts covering each phase with each part discussed separately below.

We develop a three-dimensional simulation of the Drosophila embryo, modelling the embryo as a sphere, rather than only representing a slice through the syncytium. Although the Drosophila embryo is ellipsoidal in shape a spherical shape has several computational advantages, as explained below, and due to the relatively high density of nuclei represents the global dynamics well without loss of generality. The next-neighbour relationships are defined using a Voronoi tessellation, as is usual for cell-centre based models see e.g Honda (1978); Meineke et al. (2001); Osborne et al. (2010); Schaller and Meyer-Hermann (2005). On a surface the Voronoi region $R_{i}$ can be defined for each point for the point $\mathbf{x}_{i}$ by

$$
R_{i}=\left\{\mathbf{x} \in U \mid d_{g}\left(\mathbf{x}, \mathbf{x}_{i}\right)<d_{g}\left(\mathbf{x}, \mathbf{x}_{j}\right), j \neq i\right\}
$$

where $U$ is the unit sphere and $d_{g}$ the geodesic distance. The Delaunay triangulation joins points sharing a Voronoi edge and defines the neighbour relationships. On a sphere $S$, the same neighbour relationships would be obtained using a definition based on a Euclidean distance metric and it has been shown that the Delaunay triangulation of a set of points on the surface $S$ is equivalent to the convex hull of $S$ (Sugihara (2000)), subject to the usual non-degeneracy assumptions. This has the advantage that the calculation of a convex hull is a standard computational problem, solvable in $\mathcal{O}(n \log n)$ time, where $n$ is the number of points. On non-spherical surfaces, provided the points are sufficiently dense, similar simplifications can be exploited to generate surface tilings (Fletcher et al. (2012); Trichas et al. (2012)). To restrict the nuclear motion to the embryo surface as is observed experimentally it is assumed throughout that the surface exerts a restraining force cancelling out the radial component of the internuclear force. This restraining force is in actuality actin dependent as it has been shown that inhibition of the actin cytoskeleton leads to retraction of the nuclei into the yolk region by a microtubule-based mechanism (Foe and Alberts (1983); Edgar et al. (1987)). 


\subsection{Equations of motion during interphase}

Interphase begins with the cytoskeletal networks just beginning to regrow and reconnect as mitosis comes to an end. We model the reconnection process by considering that each pair of neighbouring nuclei $i, j$ become connected and exert force on each other only when enough time has passed. To set this criterion we introduce $\bar{\tau}_{i j}=\min \left(\tau_{i}, \tau_{j}\right)$ and reconnect nuclei when $\bar{\tau}_{i j}>\alpha \tau_{\text {div }}$, see Fig. 2(a). When $\alpha<1$, the cytoskeletal networks reconnect before the period of mitosis is completed and at this point the interconnected networks dominate the dynamics.

Throughout the cell cycle the internuclear forces increase as the F-actin caps and microtubules grow and become more dense with the contact area between the caps and microtubule baskets additionally increasing over time, see Fig. 1. We thus consider a cytoskeleton network that builds the internuclear forces throughout the cell cycle up to a maximum constant internuclear force. Such a constant force law is consistent with a model for actin cap growth by polymerisation which is stalled at a constant force. It has also been recently shown that cortical nuclear organisation is myosin II independent, supporting the model that F-actin acts primarily through non-contractile filaments and does not rely on generating active forces (Kanesaki et al. (2011)). We find that this minimal model is sufficient to describe the experimental data and it is well-suited to the demands of modelling the rapidly changing cytoskeletal network and nuclear density doubling. In this model, the spatial dependencies then come about through the implicit cut-off distance generated by the time taken for reconnection, $\alpha \tau_{\text {div }}$.

We consider in the supplementary material (ESM) an alternative modelling approach based on a linear spring-based internuclear force (see Pathmanathan et al. (2009) for a comparative study of such models). We find that a spring-based model, in which the internuclear force increases with spatial distance, can reproduce the experimentally observed data only when the spring rest length is large enough that the network primarily generates repulsive internuclear forces. In this case, the profiles produced by the constant force model are recovered. We also note that care has to be taken when using spring models for the syncytium that the network does not tear apart after mitosis and generate voids in the array. Given these observations, the biological justification for constant internuclear force presented above and the fact that spring-based models introduce additional unnecessary fit parameters into the simulation we proceed with a model of a constant internuclear

force. Note that the dependence of the internuclear force on time and on the 
local directions introduced by spindle formation and mitosis distinguishes this model from the Thomson problem of minimal energy arrangements of electrons on a sphere (Bowick et al. (2002)).

The internuclear force is thus given by $\mathbf{F}_{i j}=F_{i j}\left(\mathbf{x}_{i}-\mathbf{x}_{j}\right) /\left|\mathbf{x}_{i}-\mathbf{x}_{j}\right|$ where

$$
F_{i j}\left(\bar{\tau}_{i j}\right)=\hat{F}_{\max } \begin{cases}0 & \bar{\tau}_{i j}<\alpha \tau_{\text {div }} \\ \frac{\bar{\tau}_{i j}-\alpha \tau_{\text {div }}}{(\beta-\alpha) \tau_{\text {div }}} & \alpha \tau_{\text {div }} \leq \bar{\tau}_{i j}<\beta \tau_{\text {div }} \\ 1 & \beta \tau_{\text {div }} \leq \bar{\tau}_{i j}<\gamma \tau_{c} \\ 1-\frac{\bar{\tau}_{i j}-\gamma \tau_{c}}{(1-\gamma) \tau_{c}} & \gamma \tau_{c} \leq \bar{\tau}_{i j}\end{cases}
$$

and $\hat{F}_{\max }, \beta, \gamma$ are constants (see Fig. 2(b)). To ensure a smooth transition between phases $\gamma$ is chosen to allow a gradual degradation and unless otherwise stated $\gamma=0.5$. Earlier experimental investigations suggest that both attractive and repulsive forces are present within the network, with the attractive forces being associated with the microtubule network (Kanesaki et al. (2011)). We thus here include the possibility for both attractive and repulsive forces, so that when $F_{i j}$ is positive nuclei repel each other, and when negative they attract each other.

Note that in the interconnected network nuclear neighbours interact strongly via the actin caps and microtubule baskets, see Fig. 1. Nuclear motion is predominantly resisted by this nuclear-nuclear friction rather than by the cytoplasm so that the equation of motion for the $i$ th nucleus is given, in the over-damped limit, in terms of the relative velocity

$$
\sum_{j \in n(i)}\left(\mathbf{v}_{i}-\mathbf{v}_{j}\right)=\frac{\sum_{j \in n(i)} \mathbf{F}_{i j}}{\bar{\mu}}
$$

where $\bar{\mu}$ is the effective viscosity of the two-dimensional layer of nuclei, $\mathbf{v}_{i}$ is the velocity of the $i$ th nucleus and $n(i)$ are the neighbours of $i$. The system thus behaves as a highly viscous liquid, see e.g. Bittig et al. (2008). Note that the system of equations Eq. (3) are underdetermined, and are completed by adding the condition that the centre of mass of the system is fixed in space.

\subsection{Simulating mitosis}

When the nucleus reaches the end of the cell cycle at $\tau_{i}=\tau_{c}$ the nucleus enters mitosis and divides into two daughter nuclei. In the simulation the two daughter nuclei are placed a small distance $d$ apart with the axis of division chosen randomly to represent that the spindle axis has no preferred 
(a)

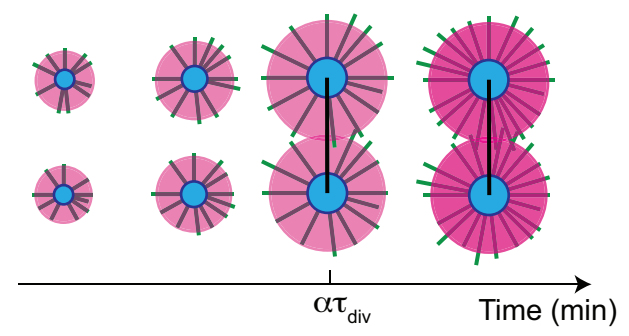

(b)

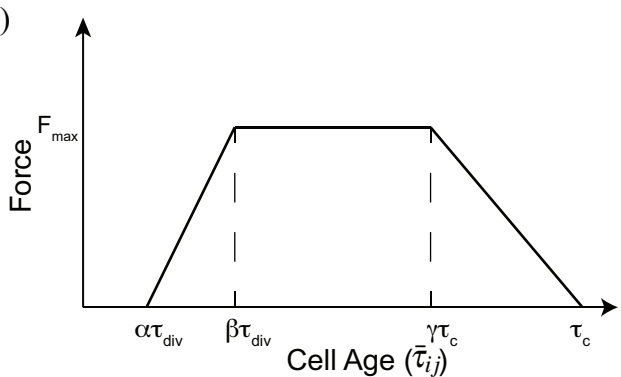

Figure 2: Interphase force laws. (a) After mitosis nuclei exert forces on each other only once the cytoskeleton has regrown sufficiently to reconnect. This takes $\alpha \tau_{\text {div }}$ minutes to achieve, where $\tau_{\text {div }}$ is the time required for mitosis. (b) Plot of internuclear force after connection. The force increases in time due to actin and microtubule growth to a maximum $\mathrm{F}_{\max }$. (Colour online).

direction as confirmed by image analysis. The mitotic phase takes time $\tau_{\text {div }}$ to be completed, during which time the daughter nuclei are driven apart by the mitotic spindle. We assume that the mitotic spindle generates a constant force $\mathbf{F}_{\text {div }}$ between the nuclei, which in this case arises due to the model of active force generation by molecular motors in which a constant number of motors work close to the stall condition (Guthardt Torres et al. (2012)). Prior to mitosis the other cytoskeletal network elements are disassembled which means that resistance to the motion of the separating nuclei is provided by the cytoplasm of the embryo rather than by internuclear friction. Thus we have as the equation of motion for nucleus $i$ at position $\mathbf{x}_{i}$ in the over-damped limit that

$$
\mathbf{v}_{i}(t)=\frac{\mathbf{F}_{\text {div }}}{\tilde{\mu}},
$$

where $\tilde{\mu}$ is the effective drag coefficient of the nucleus through the cytoplasm and $\mathbf{F}_{\text {div }}$ is the constant force between daughter nuclei. For a viscous fluid of viscosity $\mu$ and a spherical nucleus of radius $r$ Stokes' drag gives $\tilde{\mu}=6 \pi \mu r$.

During both interphase and the mitotic phase a strong repulsive hardsphere force is introduced to prevent nuclei approaching too closely to each other, however, due to the very small interaction radius used this force does not greatly contribute to the system dynamics. 


\subsection{Simulating the mitotic wave}

An additional feature of the biological system that we incorporate into the simulation is the mitotic wave. This is the small temporal offset in the start of mitosis that is observed to generate a wave of division events across the embryo. Possibly mechanisms for controlling this wave include entrainment by a guiding wave (e.g. calcium), diffusion of activating cell-cycle factors from one nucleus to another, a mechanical activation by dividing neighbouring nuclei, or synchrony by singly dividing cells. The exact biological mechanism, however, remains unclear, although a recent theoretical model by Idema et al. (2013) describing the embryo as a mechanically excitable medium suggests that mechanical signalling is an important factor. Given this current lack of knowledge, we simply introduce the phenomenon by hard-wiring it into the simulation through setting the initial age of each nucleus not to zero but rather as

$$
\tau_{i}=\frac{\tau_{c}}{v_{\text {wave }}}\left\|2 \theta_{i} / \pi-1\right\|
$$

where $\theta_{i} \in[0, \pi]$ is the polar angle illustrated in Fig. 6(c) quantifying distance along the axis of the embryo. The constant $v_{\text {wave }}$ controls the speed of the wave. Eq. (5) introduces a gradient in cell age along the embryo axis, thus generating a wave of division events. In actual fact some noise is introduced into this age profile when the simulation is initialised (see the discussion of implementation below).

\subsection{Parameter values and non-dimensionalisation}

The simulation is non-dimensionalised by rescaling time and length scales with the radius $R$ of the embryo and cell cycle time $\tau_{c}$, respectively, i.e. by introducing dimensionless lengths $\hat{r}$ and times $\hat{t}$ such that $r=R \hat{r}$ and $t=\tau_{c} \hat{t}$. The velocities are rescaled so that $v=V \hat{v}$, where $V=R / \tau_{c}$. The cell cycle length is taken as that of 13 th interphase, i.e. $\tau_{c}=21.1$ minutes (Foe and Alberts (1983)). The initial daughter separation distance is taken as $d=0.08 R$, with $\tau_{\text {div }}=0.063 \tau_{c}=1.34$ minutes (Kanesaki et al. (2011)), and the hard sphere cutoff distance $d_{\text {cut }}=0.04 R$. The time step is $0.0002 \tau_{c}$. We also introduce the non-dimensional division force $\kappa_{\text {div }}=\left\|\mathbf{F}_{\text {div }}\right\| /(\tilde{\mu} V)$ and the non-dimensional maximum ordering force $F_{\max }=\hat{F}_{\max } /(\bar{\mu} V)$, which are used to parameterise Figs. 5(b) and 6(b). The hard-sphere force is set at $3 F_{\max }$. 


\subsection{Initial conditions and implementation}

We initialise the simulation with 300 nuclei, which corresponds in terms of nuclear density to being at the beginning of interphase 12, and run the simulation for two divisions. We begin with a regular array, which is achieved by randomly distributing the nuclei and then applying a strong repulsive force. Note that as cell age is allocated using Eq. (5) to each nucleus at this initial ordering introduces a degree of noise into the distribution.

The model is implemented in $\mathrm{C}++$. To solve Eq. (3) the "PETSc" suite (Balay et al. (2011, 2010, 1997)) with an LU decomposition is used, the convex hull is calculated using "qhull" (Barber et al. (1996)), and the cell cycle model is provided by "Chaste" (Mirams et al. (2013); Pitt-Francis et al. (2009)).

\subsection{Quantification of nuclear ordering}

We consider two measures of order in the array, namely the normalised standard deviation in internuclear distances $\sigma / \mu$, which quantifies the degree of regularity in spacing, and the averaged local bond orientational order parameter $\phi$, which quantifies the deviation of the arrangement from a hexagonal lattice see e.g. Kansal et al. (2000). We choose these measures in order to compare our results with the experimental data of Kanesaki et al. (2011), in which the order of the nuclear array was tracked throughout the cell cycle in real-time. Both $\sigma / \mu$ and $\phi$ define quantifications of order that are independent of the cell density.

The parameter $\phi$ is given by

$$
\phi=\frac{1}{N} \sum_{j=1}^{N}\left|\frac{1}{n(j)} \sum_{i=1}^{n(j)} \exp \left(\mathrm{i} 6 \hat{\theta}_{i}\right)\right| .
$$

The angles $\hat{\theta}_{i}$ are the angles that the neighbours of nucleus $j$ make with a fixed reference direction, $n(j)$ is the number of neighbours of nucleus $j$ and $N$ is the total number of nuclei. For a perfect hexagonal lattice $\phi=1$, as a result of the number 6 in the argument of Eq. (6), and as the arrangement deviates from this so $\phi$ decreases. In the simulations, in order to compare with the two-dimensional data of Kanesaki et al. (2011) we project the position of each nucleus and its neighbours onto the tangent plane, with the reference direction fixed, before $\phi$ is calculated. 
(a)

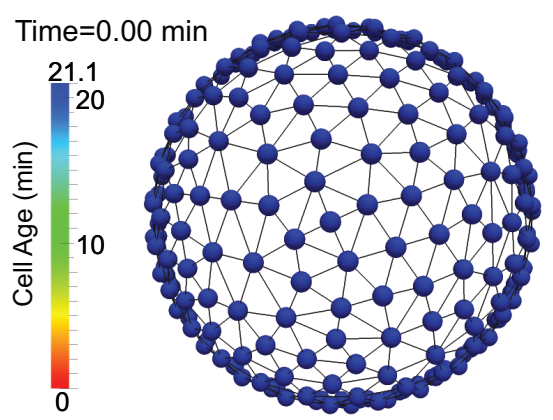

(c)

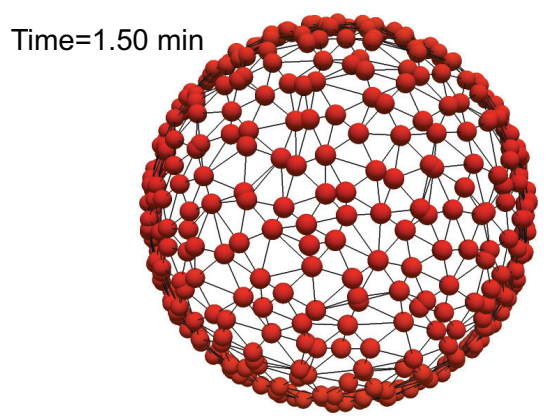

(b)

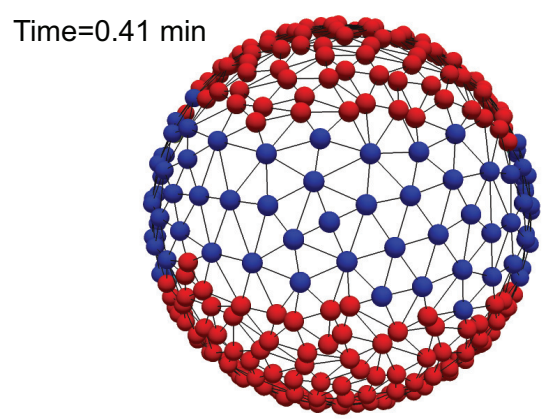

(d)

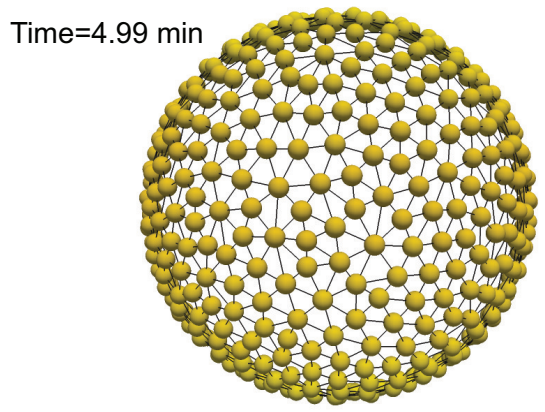

Figure 3: Snapshots of a simulation of the syncytial embryo. Starting from interphase (a) the embryo undergoes a period of mitosis (b), with nuclear division being initiated at the poles of the embryo. Subsequent to mitosis daughter nuclei are driven apart by the mitotic spindle (c) before reordering (d). Nuclear colour indicates cell age. Simulation parameters $\kappa_{\text {div }}=1.02, v_{\text {wave }}=40, \alpha=0.9, \beta=1.3, \gamma=0.5$ and $F_{\max }=7.5$. (Colour online).

\section{Results}

Simulation snapshots (Fig. 3) show that the simulation qualitatively reproduces the dynamics of nuclear self-organisation throughout the cell-cycle (compare also the simulations S2 in the supplementary material). All the distinctive visual features of the wild-type embryo have been implemented including the mitotic wave progressing from the poles to the equator of the embryo. To show that the simulation framework is able to accurately capture the dynamics of nuclear organisation in syncytial embryos we look for agreement between the simulation and experimental results. Tracking the order parameters $\sigma / \mu$ through a cell cycle we find we can achieve very good 
agreement with the experimental curves for wild type embryos at a similar stage of development, see Fig. 4(a),(b). In the case of the order parameter $\phi$, the agreement is less immediately clear due to the large variability in the experimental data, see Fig. 4(c),(d). It can, however, be seen that the experimental curves show a sharp increase in disorder during mitosis before recovering an ordered arrangement in a biphasic manner and that the simulated curves show the same qualitative behaviour with quantitative values comparable with those observed experimentally. There are two main fit parameters used to achieve this agreement, namely the strength of the mitotic spindle $\kappa_{\text {div }}$ and the reordering force parameter $F_{\max }$, the influence of each of which we consider in more detail below.

In the following, for simplicity and ease of comparison the degree of order is quantified using the more clear and robust measure of the standard deviation of the intercell distances $\sigma / \mu$ rather $\phi$.

Before proceeding, however, we note that a striking feature of the agreement between the plots is the first initial proto-peak in $\sigma / \mu$ before maximum disorder is achieved. This peak is a consequence of the onset of chromosome segregation and the instantaneous introduction of small finite distances. In the simulation these distances are introduced at division when the daughter nuclei are placed a small distance $d$ apart, with the height of the proto-peak decreasing with increasing $d$ (see supplemental Fig. S2). This makes $d$ an additional fit parameter of the system. In the experimental data, the protopeak is generated by a similar mechanism, namely the instantaneous small separations that are introduced at each time step during division through the image analysis. The effect is magnified in the simulations due to the distances introduced being in general smaller than those in the equivalent experimental images. It is interesting to see that the introducing criteria for cells being disjoint occurs equivalently in both simulation and image analysis, with similar implications for the interpretation of the data.

\subsection{Disordering is nonlinearly correlated with spindle elongation $\kappa_{\mathrm{div}}$ and is unaffected by the mitotic wave speed.}

Focusing first on the process of disordering, experimentally it is observed that immediately prior to chromosome segregation the cytoskeletal elements break down, thus allowing the mitotic spindle to drive the two daughter nuclei apart. This effect can be isolated in the simulation by including only the mitotic spindle force, switching off all other cytoskeleton-derived intercell 

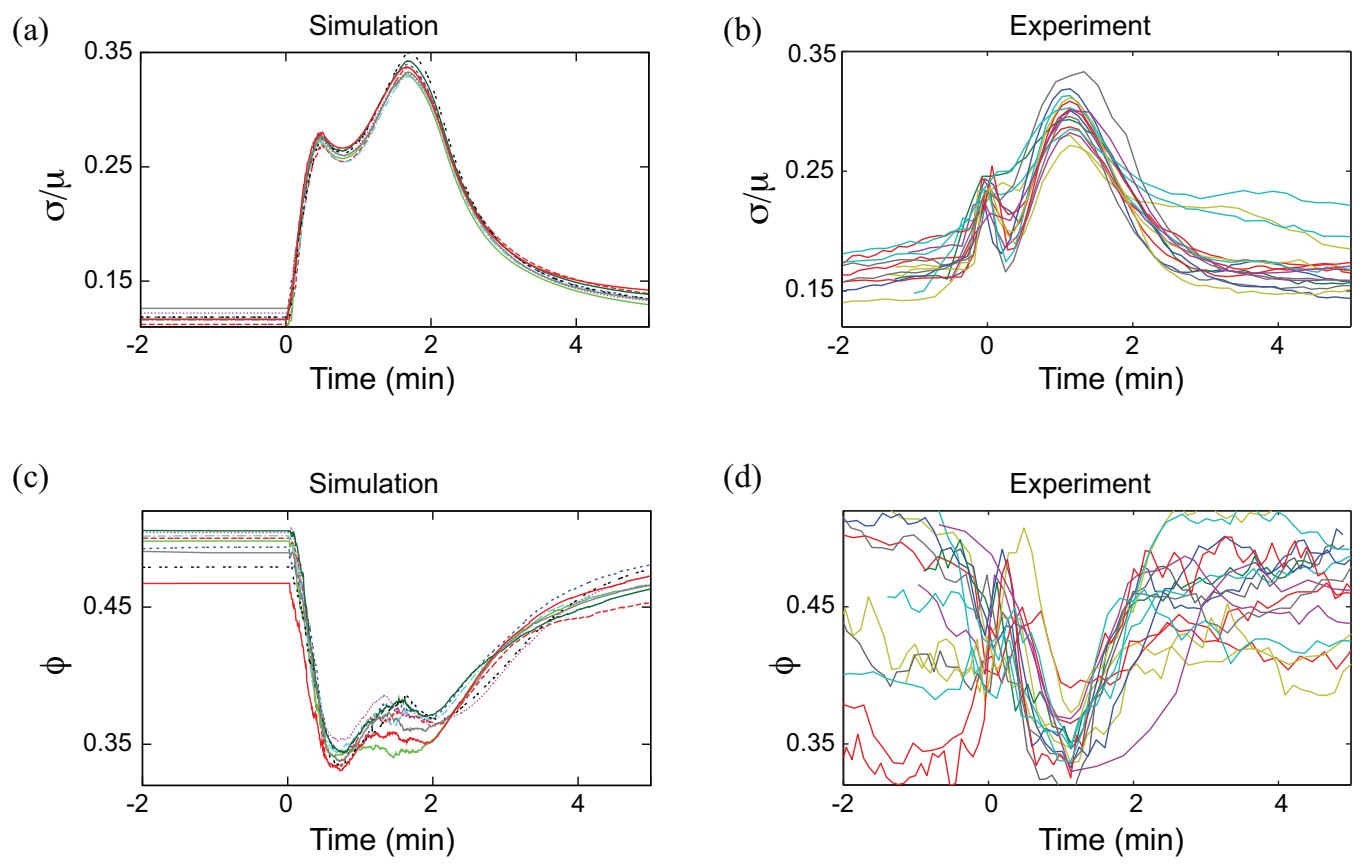

Figure 4: Quantitative comparison with experimental data of ordering in simulated embryos. The degree of order in the simulation tracked through a complete cell cycle for nine simulations compared with equivalent experimental values for Drosophila melanogaster taken from Kanesaki et al. (2011). Simulation parameters $\kappa_{\text {div }}=1.02, v_{\text {wave }}=40, \alpha=0.9$, $\beta=1.3, \gamma=0.5$ and $F_{\max }=7.5$. Normalised standard deviation of intercell distances $\sigma / \mu$ : (a) Simulated, (b) Experimental. Orientational order parameter $\phi$ : (c) Simulated, (d) Experimental. (Colour online). 

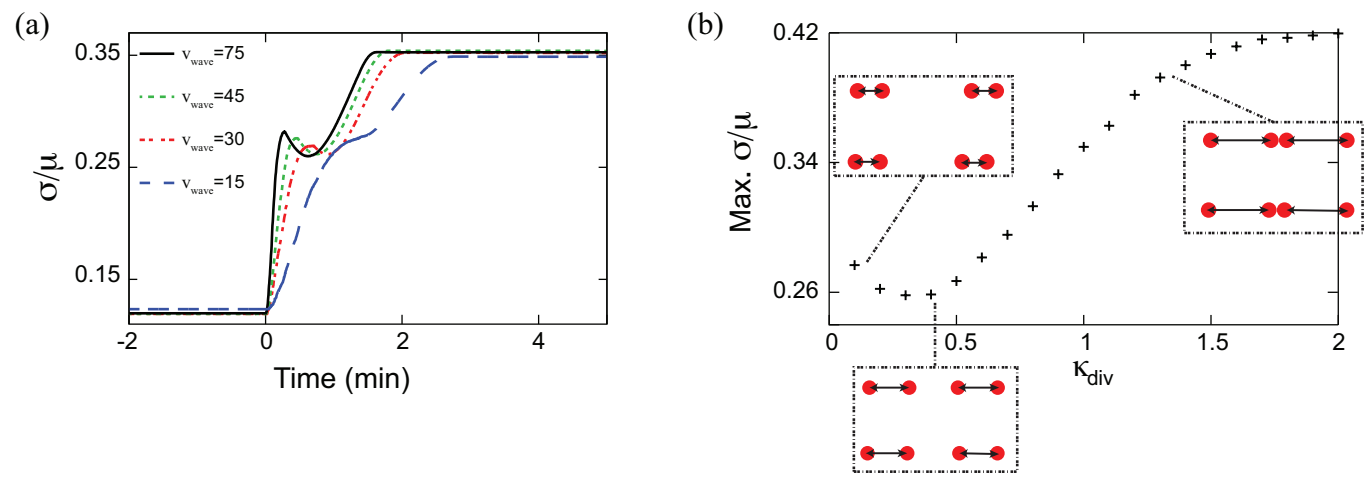

Figure 5: Disordering of syncytial embryos by mitosis. (a) Plot showing the effect of varying the mitotic wave speed on the disordering of the system. Simulation run without reordering forces, see Methods, with wave speed parameter $v_{\text {wave }}=75,45,30,15$ and $\kappa_{\text {div }}=1.02$. (b) Plot of the maximum disorder as quantified by $\sigma / \mu$ against the spindle elongation force $\kappa_{\text {div }}$, again in the absence of reordering with $v_{\text {wave }}=40$. Insets indicate the effect of increasing spindle elongation force $\kappa_{\text {div }}$ on the nuclear arrangement in a regular lattice. (Colour online).

forces, see Methods. In this case the system proceeds to disorder, but there is no reordering after the end of mitosis.

Looking first at the effect of the mitotic wave parameter $v_{\text {wave }}$ on the process of disordering, the only observed effect of a slower mitotic wave is to delay the time at which maximum disorder is achieved (see Fig. 5(a)). The maximum value of disorder and the curve shape are similar across the range of wave speeds. It has previously been reported that the maximum amount of disorder generated in the system correlates with the extension of the mitotic spindle (Kanesaki et al. (2011)). Here we see that there is a nonlinear relationship between the spindle force $\kappa_{\text {div }}$ and the observed disordering of the system (see Fig. 5(b)). The cartoons in Fig. 5(b) explain the main effect behind this nonlinearity. When the spindle is weak it can only drive the nuclei a very small distance apart thus introducing large variation in internuclear distances. Equally very strong spindles drive the nuclei a large distance apart, which again results in nuclei approaching each other closely introducing small distances into the system. Between these two extremes the spindle pushes nuclei apart but to a distance that effectively halves the inter-nuclear distances and explains the observed minimum in the graph. 


\subsection{Reordering dynamics are dependent on the reconnection time of the net-}

work and a repulsive internuclear force $F_{\max }$.

In the simulations two key parameters control the reordering dynamics of the array, namely the maximum reordering force $F_{\max }$ and the reconnection time of the nuclei after division $\alpha \tau_{\text {div }}$. To investigate the importance of reconnection timings on the ordering of the system multiple simulations were run with different values of $\alpha$, ensuring that $\beta$, which controls the rate of increase of the internuclear force post reconnection is adjusted accordingly to maintain the gradual rise in force. We find that varying $\alpha$ has a dramatic effect on the profile of the ordering curve, see Fig. 6(a). If the actin caps and the microtubule baskets connect nuclei too late then a characteristic flattened top is introduced into profile of the order parameter $\sigma / \mu$. Such a plateau has not been observed experimentally. Interestingly the criterion to remove this feature is that $\alpha \leq 1$, i.e. that the cytoskeletal elements have begun to reconnect nuclei before the mitotic phase is completed. This has the added implication that the cytoskeletal networks nearer the poles will reconnect nuclei before all nuclei have exited mitosis in the embryo.

The reordering of the nuclei subsequent to mitosis is driven by the reordering force. Fig. 6(b) clearly shows that attractive forces are insufficient to generate system order post mitosis. When $F_{\max }$ is negative the degree of system disorder is observed to increase subsequent to the division phase. This increase can be ascribed to the tendency of attractive forces to induce clustering of nuclei within the embryo.

In contrast for strong repulsive forces system order is dynamically recovered in a manner comparable to that experimentally observed. The strength of the maximum repulsive force has a strong effect on the qualitative dynamics of system ordering. In Fig. 6(b), $\sigma / \mu$ is plotted for a range of repulsive forces ranging from 0 to 10 are plotted. For weaker forces it is observed that the initial steep recovery is lost although the system does still recover over a much longer timescale, until at $F_{\max }=0$ the system fails to reorder.

Taken together these results show that these repulsive forces (changing in time in coordination with the cell-cycle) are necessary and sufficient to drive the ordering of the embryo after mitosis, with a strong maximum repulsive force required to generate the observed biphasic reordering dynamics. It is thus also clear that any attractive forces present in the system must be of a smaller magnitude than the main repulsive forces that drive ordering of the embryo. 
(a)

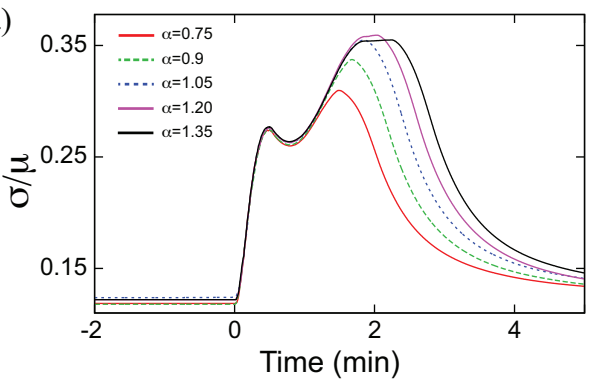

(b)

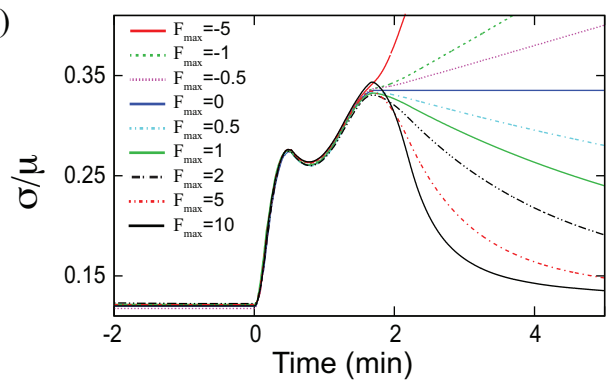

(c)

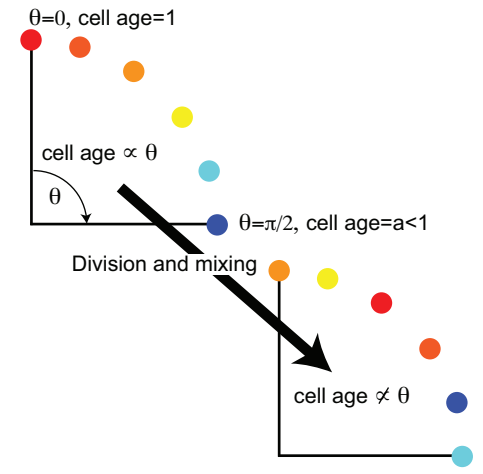

(d)

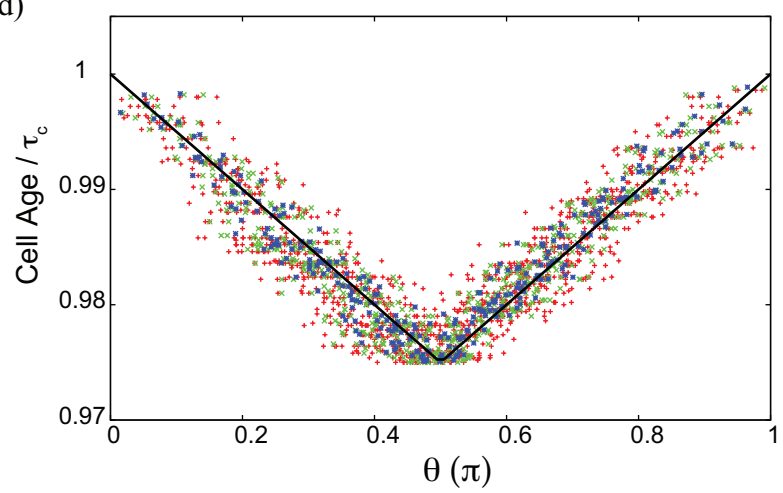

Figure 6: Recovery of positional order after mitosis. (a) Effect of $\alpha$ on reordering. $\alpha=$ $0.75,0.9,1.05,1.20,1.35$ with $\beta=1.15,1.30,1.45,1.60,1.75$, respectively and with $\mathrm{F}_{\max }=$ 7.5. ( $\beta$ is chosen to keep the slope of $F$ constant). (b) Effect of $F_{\max }$ on reordering. $\mathrm{F}_{\max }=-5$ to 10 from top to bottom with $\alpha=0.9, \beta=1.3$. (c) Schematic showing the initial relationship between cell age and the azimuthal angle $\theta$, with a possible arrangement post division. (d) Graph of cell age against $\theta$, for the initial arrangement with noise added (blue stars), after one division (green crosses) and after a second division (red plus signs), with $F_{\max }=7.5, \alpha=0.9, \beta=1.3$. (Colour online). The straight line is the perfect unmixed arrangement Eq. (5). $\left(\kappa_{\mathrm{div}}=1.02, v_{\text {wave }}=40, \gamma=0.5\right.$ for all simulations $)$. 


\subsection{Nuclei maintain their relative positions through cell cycles.}

A further important observation that can be made from the simulations is that there is very little mixing amongst the nuclei following mitosis, with daughter nuclei in general maintaining their positions relative to the poles of the embryo. At the start of the simulation each nucleus is given an age that is dependent on their position relative to the poles, with nuclei at the poles more mature and closer to division. Cell age then linearly decays with azimuthal angle $\theta$ so that nuclei at the equator are younger and divide last (see Fig 6(c)).

This temporal offset is inherited by daughter nuclei after division. Cell division and rearrangement act to mix the nuclei after each division and the linear relationship between cell age and azimuthal angle will be lost over time, finally obtaining an arrangement with random positioning (Fig 6(c)). A plot of cell age against $\theta$ over two cell divisions is shown in Fig 6(d). The blue star symbols are the initial distribution of ages into which noise has been introduced just before the first division takes place (see Methods), the green crosses are the positions just before the second division and the red pluses are the positions at the end of the final interphase. A linear relationship between cell age and $\theta$ is strikingly maintained across these two cell cycles with a slight increase in the breadth of the distribution at each division. This observation is of interest in that it suggests that control of the mitotic wave could be achieved at least partially through templating the wave profile early in the developmental process, and that this inheritance property could act to stabilise the wave through cell cycles.

\section{Discussion}

We have presented here individual cell-centre based simulations of the dynamics of syncytial embryos, and have shown that our model can describe the observed ordering dynamics of nuclei throughout the cell cycle. An unusual aspect of this system is the rapidity of development, with almost synchronous nuclear division occurring within a fixed geometry, which imposes constraints on the potential internuclear forces. We have shown that simple force laws with a constant repulsive order force are sufficient to reproduce the experimental data. The temporal changes in the cytoskeletal networks are of crucial importance to the system dynamics and we find that it is necessary for the cytoskeletal networks to quickly reform before anaphase is everywhere 
completed (i.e. $\alpha \leq 1$ ). Provided that $\alpha \leq 1$, we can achieve good quantitative agreement between our simulations and the reported experimental data using only the two key fit parameters $\kappa_{\text {div }}$, which quantifies the strength of the mitotic spindle, and $F_{\max }$, the maximum reordering force. The mitotic spindle force $\kappa_{\text {div }}$ nonlinearly correlates to disordering and within the usual physiologically regimes we see that increasing the strength of the spindle increases system disorder. Increasing the reordering repulsion $F_{\max }$ controls how quickly we are able to achieve a regular array post mitosis. We note that the need for strong repulsive forces for correct system ordering was also observed when using a network of interconnected springs, suggesting a conserved mechanism of action. The need for repulsive forces for ordering is similar, but in detail different from, the situation with inert particles like colloids (Bowick et al. (2002)). The essential difference is that here we deal with a dynamic system that completely changes its character during mitosis, when the cytoskeleton networks disassemble and the mitotic spindle appears as a local and anisotropic perturbation. It is remarkable that we find that the fast recovery of repulsive interactions is still sufficient to keep order in this system.

Experimental studies into the individual roles of the F-actin and microtubules usually attempt to isolate their functions by the use of e.g. mutant embryos and microinjection of drugs to disrupt the cytoskeleton (Cao et al. (2010)). Looking at the recently reported quantitative data on ordering in Kanesaki et al. (2011), the injection of the drug latrunculin, which depolymerises F-actin while leaving the microtubules intact, results in an increase of disordering in the system in a manner strikingly consistent with Fig. 6(b) with $F_{\max }$ attractive. Our simulations thus support the reported suggestion that the microtubule network acts attractively throughout the embryo but that these forces are usually screened out by the actin caps. The emerging model of an attractive tensile network of microtubules, resisted by actin caps bearing the tensional forces, has clear analogies with the tensegrity model of cells (Ingber (2003); Stamenović and Ingber (2009)). Cellular tensegrity models consider that the actomyosin complexes of the cell generate a prestress resisted by microtubules acting as compression 'struts' and by cellular attachments to the ECM. This antagonistic force mechanism enables the cell to stabilise its shape and resist deformation. We note that here we encounter a similar situation, but with reversed roles of the actin networks and microtubules. Although in principle not necessary for generating system order in the syncytial embryo, microtubule-associated attractive forces could play a 
key role in stabilising the system and reducing spatial noise.

A particular advantage of using simulations to investigate biological systems is the ability to easily access parameter regimes not experimentally achievable. For example, in this study we have studied the roles of the mitotic wave and mitotic spindle in system disordering by varying both the wave speed and the strength of the mitotic spindle in a controlled manner. Our investigation of the role of the mitotic spindle in generating disorder showed a nonlinear relationship between spindle strength and disorder. This had not previously been noticed as the small parameter regime that was experimentally accessible indicated a nearly linear relationship (Kanesaki et al. (2011)).

The simulations model the reordering force as independent of internuclear spacing, although increasing in time. Such a force law is consistent with the fact that the actin caps grow by polymerisation and are stalled at constant force, although it is not possible at this stage to distinguish between different force generation models. To further develop the model and establish in detail the specifics of the internuclear forces requires more detailed quantitative experimental data. In particular, experiments are required that mechanically perturb the network in a focused manner, for example, the use of laser ablation to carefully cut out sections of the cytoskeletal network. Within the simulation framework, such experiments could be reproduced by removing neighbour connections from the triangulation. An additional challenge, is to obtain data in three dimensions at high temporal resolution rather than using the two-dimensional slices usually considered. There have been studies looking at the entire embryo in three dimensions on fixed embryos (Keränen et al. (2006)), but an exciting development is the recent interest in using imaging and data analysis techniques to track live nuclei in real-time in three dimensions (Krzic et al. (2012); Tomer et al. (2012)).

Despite allowing isotropic division and free nuclear movement very little mixing of nuclei is observed across the cell cycles. Daughter nuclei thus inherit and in general maintain their spatial position relative to the poles as indeed observed experimentally (Krzic et al. (2012)). Although this might to some degree be expected due to the energy barrier imposed by the repulsive internuclear forces, this observation has profound implications for the system. It suggests that templating could play a role in maintaining the mitotic wave itself, as once the temporal offset is set it can be maintained over several cell divisions. It would be interesting to use the simulation to investigate the possible mechanisms of control of the mitotic wave and its interplay with 
the ordering dynamics. Of particular interest in this context is the recent work of Idema et al. (2013) modelling the syncytium and mitotic wave as a mechanically excitable medium. However, such an approach would not only require a more detailed mechanical model, but also more experimental input on the details of the underlying regulation, including the signaling proteins regulating the regrowth of the actin network.

We note that mitosis is a critical moment for the mechanical stability in the syncytium and that by using a wave, the system distributes this instability in space and time; as only a small region of the whole syncytium is dividing at a given time, there is no global instability. The maintenance of relative nuclear positioning could be significant for morphogen gradient establishment and embryo segmentation. The gap genes hunchback, giant, Krüppel and knirps, in particular, pattern the trunk region of the embryo early in development into regions with clearly defined boundaries (Jaeger (2011)). This restriction of mixing by physical forces in the syncytium echoes that observed at later stages of development. For example, in the Drosophila wing disc, Aliee et al. (2012) have shown using computational modelling combined with experiment that acto-myosin generated cell tension maintains compartment boundaries in the face of cellular proliferation. Our simulation framework now enables a similar combined approach to be undertaken at the syncytial stage, elucidating how biochemical and mechanical signalling coordinate in early development.

\section{Acknowledgments}

CMD and TK were supported by the Center for Modelling and Simulation in the Biosciences (BIOMS) and a ZMBH post-doctoral fellowship, respectively. JG acknowledges support by the German Research Council. USS is a member of the cluster of excellence CellNetworks at the University of Heidelberg.

Aliee, M., Röper, J.C., Landsberg, K.P., Pentzold, C., Widmann, T.J., Jülicher, F., Dahmann, C., 2012. Physical mechanisms shaping the Drosophila dorsoventral compartment boundary. Curr. Biol. 22, 967-976.

Balay, S., Brown, J., , Buschelman, K., Eijkhout, V., Gropp, W.D., Kaushik, D., Knepley, M.G., McInnes, L.C., Smith, B.F., Zhang, H., 2010. PETSc Users Manual. Technical Report ANL-95/11 - Revision 3.1. Argonne National Laboratory. 
Balay, S., Brown, J., Buschelman, K., Gropp, W.D., Kaushik, D., Knepley, M.G., McInnes, L.C., Smith, B.F., Zhang, H., 2011. PETSc web page. http://www.mcs.anl.gov/petsc.

Balay, S., Gropp, W.D., McInnes, L.C., Smith, B.F., 1997. Efficient management of parallelism in object oriented numerical software libraries, in: Arge, E., Bruaset, A.M., Langtangen, H.P. (Eds.), Modern Software Tools in Scientific Computing, Birkhäuser Press. pp. 163-202.

Barber, C., Dobkin, D., Huhdanpaa, H., 1996. The quickhull algorithm for convex hulls. ACM T Math. Software 22, 469-483.

Bittig, T., Wartlick, O., Kicheva, A., González-Gaitán, M., Jülicher, F., 2008. Dynamics of anisotropic tissue growth. New J. Phys. 10, 063001-1063001-10.

Blankenship, J.T., Wieschaus, E., 2001. Two new roles for the Drosophila AP patterning system in early morphogenesis. Development 128, 5129-5138.

Bollenbach, T., Pantazis, P., Kicheva, A., Bokel, C., González-Gaitán, M., Jülicher, F., 2008. Precision of the Dpp gradient. Development 135, 11371146 .

Bornens, M., 2008. Organelle positioning and cell polarity. Nat. Rev. Mol. Cell Bio. 9, 874-886.

Bowick, M., Cacciuto, A., Nelson, D., A., T., 2002. Crystalline order on a sphere and the generalized Thomson problem. Phys. Rev. Lett. 89, 185502.

Cao, J., Crest, J., Fasulo, B., Sullivan, W., 2010. Cortical actin dynamics facilitate early-stage centrosome separation. Curr. Biol. 8, 770-776.

Drasdo, D., 2005. Coarse graining in simulated cell populations. Adv. Complex Syst. 8, 319-363.

Drasdo, D., Höhme, S., 2005. A single-cell-based model of tumor growth in vitro: monolayers and spheroids. Phys. Biol. 2, 133-147.

Edgar, B.A., Odell, G.M., Schubiger, G., 1987. Cytoarchitecture and the patterning of fushi tarazu expression in the Drosophila blastoderm. Genes Dev. 1, 1226-1237. 
Fletcher, A.G., Breward, C.J.W., Chapman, S.J., 2012. Mathematical modeling of monoclonal conversion in the colonic crypt. J. Theor. Biol. 300, $118-133$.

Fletcher, D.A., Mullins, R.D., 2010. Cell mechanics and the cytoskeleton. Nature 463, 485-492.

Foe, V.E., Alberts, B.M., 1983. Studies of nuclear and cytoplasmic behaviour during the five mitotic cycles that precede gastrulation in Drosophila embryogenesis. J. Cell Sci. 61, 31-70.

Foe, V.E., Field, C.M., Odell, G.M., 2000. Microtubules and mitotic cycle phase modulate spatiotemporal distributions of F-actin and myosin II in Drosophila syncytial blastoderm embryos. Development 127, 12767-1787.

Foe, V.E., Odell, G.M., Edgar, B.A., 1993. Development of Drosophila melanogaster. Cold Spring Harbor Press. chapter Mitosis and morphogenesis in the Drosophila embryo: Point and Counterpoint. pp. 149-300.

Guthardt Torres, P., Bischofs, I.B., Schwarz, U.S., 2012. Contractile network models for adherent cells. Phys. Rev. E 85, 011913.

Honda, H., 1978. Description of cellular patterns by Dirichlet domains: The two-dimensional case. J. Theor. Biol. 72, 523 - 543 .

Idema, T., Dubuis, J.O., Kang, L., Manning, M., Nelson, P., Lubensky, T.C., Liu, A., 2013. The syncytial Drosophila embryo as a mechanically excitable medium. PLoS ONE 8, e77216.

Ingber, D.E., 2003. Tensegrity I. Cell structure and hierarchical systems biology. J. Cell Sci. 116, 1157-1173.

Jaeger, J., 2011. The gap gene network. Cell. Mol. Life Sci. 68, 243-274.

Kanesaki, T., Edwards, C.M., Schwarz, U.S., Grosshans, J., 2011. Dynamic ordering of nuclei in syncytial embryos: a quantitative analysis of the role of cytoskeletal networks. Integr. Biol. 3, 112-119.

Kansal, A.R., Truskett, T.M., Torquato, S., 2000. Nonequilibrium hard-disk packings with controlled orientational order. J. Chem. Phys. 113, 48444851. 
Keränen, S.V.E., Fowlkes, C.C., Luengo Hendriks, C.L., Sudar, D., Knowles, D.W., Malik, J., Biggin, M.D., 2006. Three-dimensional morphology and gene expression in the Drosophila blastoderm at cellular resolution II: dynamics. Genome Biol. 7, R124. doi:10.1186/gb-2006-7-12-r124.

Krzic, U., Gunther, S., Saunders, T.E., Streichan, S.J., Hufnagel, L., 2012. Multiview light-sheet microscope for rapid in toto imaging. Nature Methods $9,730-733$.

Ma, D., Amonlirdviman, K., Raffard, R.L., Abate, A., Tomlin, C.J., Axelrod, J.D., 2008. Cell packing influences planar cell polarity signaling. Proc Natl Acad Sci U S A 105, 18800-18805. doi:10.1073/pnas.0808868105.

Mazumdar, A., Mazumdar, M., 2002. How one becomes many: blastoderm cellularization in Drosophila melanogaster. Bioessays 24, 1012-1022.

Meineke, F.A., Potten, C.S., Loeffler, M., 2001. Cell migration and organization in the intestinal crypt using a lattice-free model. Cell Prolif. 34, 253-266.

Metzger, T., Gache, V., Xu, M., Cadot, B., Folker, E., Richardson, B.E., Gomes, E.R., Baylies, M.K., 2012. MAP and kinesin-dependent nuclear positioning is required for skeletal muscle function. Nature 484, 120-124.

Mirams, G.R., Arthurs, C.J., Bernabeu, M.O., Bordas, R., Cooper, J., Corrias, A., Davit, Y., Dunn, S.J., Fletcher, A.G., Harvey, D.G., Marsh, M.E., Osborne, J.M., Pathmanathan, P., Pitt-Francis, J., Southern, J., Zemzemi, N., Gavaghan, D.J., 2013. Chaste: An open source C++ library for computational physiology and biology. PLoS Comput. Biol. 9, e1002970.

Murray, P.J., Edwards, C.M., Tindall, M.J., Maini, P.K., 2012. Classifying general nonlinear force laws in cell-based models via the continuum limit. Phys. Rev. E 85, 021921.

Osborne, J., Walter, A., Kershaw, S., Mirams, G., Fletcher, A., Pathmanathan, P., Gavaghan, D., Jensen, O., Maini, P., Byrne, H., 2010. A hybrid approach to multi-scale modelling of cancer. Philos. Trans. R. Soc. London, Ser. A 368, 5013-5028. 
Pathmanathan, P., Cooper, J., Fletcher, A., Mirams, G., Murray, P., Osborne, J., Pitt-Francis, J., Walter, A., Chapman, S.J., 2009. A computational study of discrete mechanical tissue models. Phys. Biol. 6, 036001.

Pitt-Francis, J., Pathmanathan, P., Bernabeu, M.O., Bordas, R., Cooper, J., Fletcher, A.G., Mirams, G.R., P., M., Osborne, J.M., Walter, A., Chapman, S.J., Garny, A., van Leeuwen, I.M.M., Maini, P.K., Rodrigez, B., Waters, S.L., Whiteley, J.P., Byrne, H.M., Gavaghan, D.J., 2009. Chaste: A test-driven approach to software development for biological modelling. Comp. Phys. Comm. 180, 2452-2471.

Schaller, G., Meyer-Hermann, M., 2005. Multicellular tumor spheroid in an off-lattice voronoi-delaunay cell model. Phys. Rev. E 71, 051910.

Schejter, E.D., Wieschaus, E., 1993. Functional elements of the cytoskeleton in the early Drosophila embryo. Annu. Rev. Cell Biol. 9, 67-99. doi:10.1146/annurev.cb.09.110193.000435.

Stamenović, D., Ingber, D.E., 2009. Tensegrity-guided self assembly: from molecules to living cells. Soft Matter 5, 1137-1145.

Sugihara, K., 2000. Three-dimensional convex hull as a fruitful source of diagrams. Theor. Comput. Sci. 235, 325-337.

Tomer, R., Khairy, K., Keller, P.J., 2012. Quantitative high-speed imaging of entire developing embryos with simultaneous multiview light-sheet microscopy. Nature Methods 9, 755-763.

Trichas, G., Smith, A.M., White, N., Wilkins, V., Watanabe, T., Moore, A., Joyce, B., Sugnaseelan, J., Rodriguez, T.A., Kay, D., Baker, R.E., Maini, P.K., Srinivas, S., 2012. Multi-cellular rosettes in the mouse visceral endoderm facilitate the ordered migration of anterior visceral endoderm cells. PLoS Biology 10, e1001256. 